\title{
Functional Dissociation of the Frontoinsular and Anterior Cingulate Cortices in Empathy for Pain
}

\author{
Xiaosi Gu, ${ }^{1,2}$ Xun Liu, ${ }^{1}$ Kevin G. Guise, ${ }^{1,2}$ Thomas P. Naidich, ${ }^{3}$ Patrick R. Hof, ${ }^{4}$ and Jin Fan ${ }^{1,4}$ \\ ${ }^{1}$ Department of Psychiatry, ${ }^{2}$ Graduate School of Biological Sciences, and Departments of ${ }^{3}$ Radiology and ${ }^{4}$ Neuroscience, Mount Sinai School of Medicine, \\ New York, New York 10029
}

The frontoinsular cortex (FI) and the anterior cingulate cortex (ACC) are thought to be involved in empathy for others' pain. However, the functional roles of FI and ACC in empathetic responses have not yet been clearly dissociated in previous studies. In this study, participants viewed color photographs depicting human body parts in painful or nonpainful situations and performed either pain judgment (painful/ nonpainful) or laterality judgment (left/right) of the body parts. We found that activation of FI, rather than ACC, showed significant increase for painful compared with nonpainful images, regardless of the task requirement. Our data suggest a clear functional dissociation between FI and ACC in which FI is more domain-specific than ACC when processing empathy for pain.

\section{Introduction}

Empathy, the ability to understand and echo other people's sensory and emotional states, enables us to relate to one another and form social relationships. Recent studies on neural mechanisms of empathy for pain (Singer et al., 2004; Jackson et al., 2005; Gu and Han, 2007), disgust (Wicker et al., 2003), and pleasant experiences (Jabbi et al., 2007) indicate that empathy is subserved by a cortical network primarily consisting of the frontoinsular cortex (FI) and the anterior cingulate cortex (ACC). FI and ACC are coactivated in virtually all neuroimaging studies on pain (Peyron et al., 2000; Wager et al., 2004) and empathy for pain (Singer et al., 2004; Jackson et al., 2005; Gu and Han, 2007). Activation levels of these two regions correlate with subjective ratings of the unpleasantness of pain and dispositional measures of empathy, suggesting that both regions subserve the affective-motivational rather than the sensory-discriminative aspect of pain (Singer et al., 2004; Jackson et al., 2005).

Historically, FI has been considered a limbic sensory region responsible for polymodal sensory integration, representation of visceral responses (Critchley, 2004; Critchley et al., 2004), and conscious awareness of bodily sensations and subjective feelings (Craig et al., 2000). In contrast, ACC is known as a limbic motor cortex that participates in voluntary control of multiple domains of behaviors, such as self-initiated behaviors (Cohen et al., 1999), pain (Davis et al., 1994; Rainville et al., 1997), emotion and personality (Cohen et al., 2001), learning the value of actions (Kennerley et al., 2006), and social interaction (Rudebeck et al., 2006).

Received Sept. 29, 2009; revised Dec. 8, 2009; accepted Jan. 22, 2010.

This research was supported by National Institutes of Health (NIH) Grant MH083164 (to J.F.), National Center for Research Resources (NCRR) Grant M01 RR00071, and James S. McDonnell Foundation Grant 22002078 (to P.R.H.). Its contents are solely the responsibility of the authors and do not necessarily represent the official views of the NIH or NCRR. The funders had no role in study design, data collection and analysis, decision to publish, or preparation of this manuscript. We thank Dr. Michael I. Posner for helpful comments.

Correspondence should be addressed to Jin Fan, Laboratory of Neuroimaging, Department of Psychiatry, Mount Sinai School of Medicine, One Gustave L. Levy Place, Box 1230, New York, NY 10029. E-mail: Jin.Fan@mssm.edu. DOI:10.1523/JNEUROSCI.4844-09.2010

Copyright $\odot 2010$ the authors $\quad 0270-6474 / 10 / 303739-06 \$ 15.00 / 0$
It is likely that bodily states are generated in ACC and mapped back as feelings in the insula.

Although it is widely accepted that both FI and ACC are coactivated in processing empathy for others' pain, and much of the research has focused on the commonality of FI and ACC, it remains unclear why such structurally distinct regions appear functionally inseparable and what distinct role each plays in cognitive processes such as empathy for pain. The current study aimed to investigate the specific roles of FI and ACC involved in empathy for others' pain using a modified empathy paradigm (Jackson et al., 2005; Gu and Han, 2007) in which subjects viewed color photographs depicting other people's hands and feet in painful or nonpainful (neutral) situations (see Fig. 1). Because FI and ACC activation is usually correlated with task difficulty (Naito et al., 2000; Hahn et al., 2007; Eckert et al., 2009), the cognitive load of experimental conditions were carefully matched in our paradigm. We hypothesized that FI, rather than ACC, would play a more specific role in empathetic processing of pain and show empathy-specific activation in response to photographs depicting painful situations compared with nonpainful photographs, regardless of the task requirement as long as the tasks enlist equivalent cognitive load. We also hypothesized that empathetic responses processed in FI can be elicited automatically without explicit cognitive demand to empathize with another individual.

\section{Materials and Methods}

Subjects. Eighteen healthy adults (nine women; 22-28 years old, mean age of 24.8 years) participated in the study. All subjects were right-handed, had normal color vision, and reported no previous or current psychiatric or neurological conditions. Subjects were informed of the study requirements and provided written consent before participation. The study was approved by the Institutional Review Board of Mount Sinai School of Medicine (MSSM; New York, NY).

Stimuli and procedure. Experimental stimuli included a set of 216 digital color photographs showing another person's left or right hand or foot in painful or nonpainful situations that are similar to the stimuli used in previous studies (Jackson et al., 2005; Gu and Han, 2007). There were 54 photographs (30 foot photos and 24 hand photos) in each of the four 


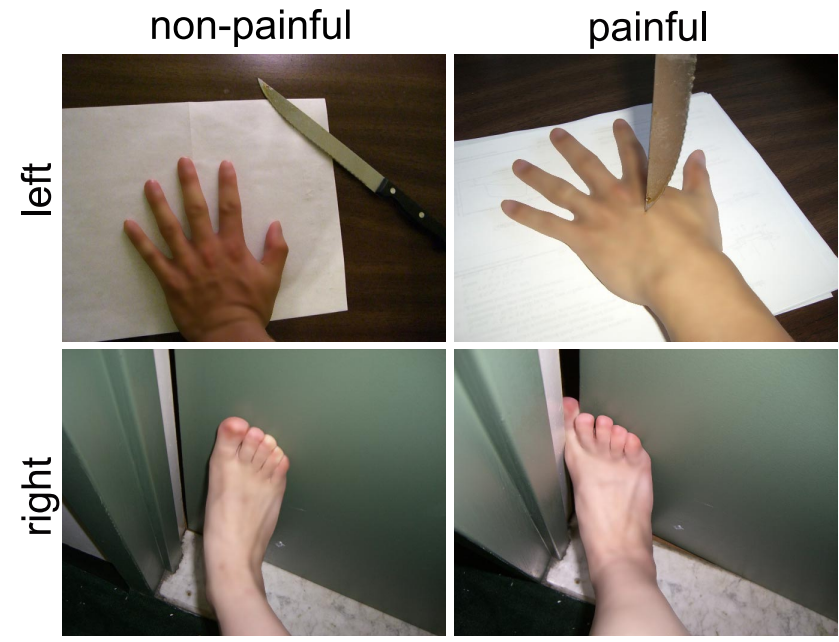

Figure 1. Sample stimuli of the experimental stimuli set of 216 digital color photographs showing another person's left or right hand or foot in painful or nonpainful situations. Each stimulus was displayed for $2500 \mathrm{~ms}$ followed by a fixation of $1500 \mathrm{~ms}$. Subjects were asked to choose between "nonpainful" and "painful" for the TP and between "left" and "right" for the TL through button press.

categories (painful-left, painful-right, nonpainful-left, nonpainful-right) (Fig. 1). The photographs depicted incidents that may happen in everyday life and were taken from the first-person perspective so that subjects would not have to perform mental rotation before judging pain or laterality. The images were slightly blurred with a Gaussian filter to remove any gender or age bias. Half of the photographs showed painful events and the other half showed nonpainful events that were identical in physical properties (i.e., context, brightness, contrast). The photographs were rated by an independent group of 30 subjects based on a 5-point scale from 1 ("not painful at all") to 5 ("extremely painful"). The pain ratings of painful and nonpainful photographs ( $3.5 \pm 0.7$ and $1.1 \pm 0.2$, respectively) were significantly different $\left(t_{(29)}=20.73, p<0.001\right)$. The tasks were chosen from a variety of cognitive tasks based on their cognitive load indexed by reaction time (RT) and accuracy from a pilot behavioral study.

An event-related design was used. There were a total of four runs. In two runs, subjects were instructed to judge whether the person in the photograph was suffering from pain or not [task pain (TP)]; in the other two runs, they were told to judge the laterality of the hand/foot [task laterality (TL)]. The order of the four runs (two TP runs and two TL runs) was counterbalanced between subjects. Each run included 27 trials of painful photographs and 27 trials of nonpainful photographs. In addition, 27 null trials of blank screen with a fixation in the center were included to jitter the intertrial intervals. The order of trials (including null trials) in each run was simulated to determine the optimal randomization for design efficiency (http://afni.nimh.nih. gov/pub/dist/HOWTO/howto/ht03_stim). Such manipulation prioritized the detection of stimulus-related effects that were the main effects of interest. This yielded a 2 (task: TP vs TL) $\times 2$ (stimulus: painful vs nonpainful) factorial design with four experimental conditions (TP-painful, TP-nonpainful, TL-painful, TL-nonpainful). Each photograph was displayed for $2500 \mathrm{~ms}$ followed by a fixation of $1500 \mathrm{~ms}$. Subjects were instructed to respond as quickly and accurately as possible.

Functional MRI data acquisition and analysis. All MRI data were obtained on a 3 T Siemens Allegra MRI system at MSSM. Foam padding was used to keep the subject's head still. All images were acquired along axial planes parallel to the anterior commissure-posterior commissure (AC$\mathrm{PC})$ line. A high-resolution, $\mathrm{T}_{2}$-weighted anatomical volume of the whole brain was acquired on an axial plane parallel to the AC-PC line with a turbo spin-echo pulse sequence. The functional MRI (fMRI) imaging was performed using a gradient-echo echo-planar imaging (EPI) sequence using the following protocol: 40 axial slices, $4 \mathrm{~mm}$ thick, skip = $0 \mathrm{~mm}$, repetition time $=2500 \mathrm{~ms}$, echo time $=27 \mathrm{~ms}$, flip angle $=82^{\circ}$, field of view $=240 \mathrm{~mm}$, and matrix size $=64 \times 64$. Slices were obtained corresponding to the $\mathrm{T}_{2}$-weighted anatomical images. Four series of EPIs corresponding to the four runs were acquired. Each series started with two dummy volumes before the onset of the task to allow for equilibration of $\mathrm{T}_{1}$ saturation effects, followed by 154 image volumes for each run.

Event-related analyses of the fMRI data from the two tasks were conducted using statistical parametric mapping (SPM) (SPM5; Wellcome Trust Centre for Neuroimaging, London, UK). The functional scans were adjusted for slice timing, realigned to the mean volume, coregistered to the $T_{2}$ image, normalized to a standard template (Montreal Neurological Institute), and spatially smoothed with an $8 \times 8 \times 8 \mathrm{~mm}$ full-width-at-half-maximum Gaussian kernel. General linear modeling (Friston et al., 1995) was then conducted for the functional scans from each participant by modeling the observed event-related blood oxygenation level-dependent signals. Regressors were created by convolving a train of delta functions representing the sequence of individual events with the default SPM basis function, which consists of a synthetic hemodynamic response function composed of two gamma functions (Friston et al., 1998). There were four [2 (TP, TL) $\times 2$ (painful, nonpainful) $]$ regressors. Six parameters generated during motion correction were entered as covariates. Linear contrasts of the parameter estimates were made to identify the main effects of task and stimulus and the interaction effect between task and stimulus, resulting in images of contrast estimate for these effects of each participant. These images from all participants were entered into a second-level group analysis conducted with a random-effects statistical model. Significant activations related to effects of interest were identified with voxelwise $p$ value exceeding 0.05 , corrected for false discovery rate (Genovese et al., 2002) in conjunction with clusterwise $p$ value exceeding 0.05 uncorrected, to control for regional effects and represent topographical inferences (resampled as $2 \times 2 \times 2 \mathrm{~mm}$ ).

To define regions of interest (ROIs), we first compared all four experimental conditions against baseline fixation. The contrast of all (combined) conditions versus baseline was used for localizing ROIs (as opposed to a combination of individual conditions each compared vs baseline which would be biased) because this contrast is orthogonal to the effects of interest. Spherical ROIs of $6 \mathrm{~mm}$ radius were created using the MarsBaR toolbox (http://marsbar.sourceforge.net) for ACC [posterior rostral cingulate zone; Fan et al. (2008)] and bilateral FI (mean parameter estimates were averaged across left and right FI). Parameter estimates were extracted from each ROI for each of the four experimental conditions from each subject using MarsBaR and then entered into an ANOVA. This subsequent ANOVA is independent from previous contrasts because each contrast in the ANOVA is orthogonal to the main effect of all conditions versus baseline. Separate psychophysiological interaction (PPI) analyses were also performed using FI or ACC as the seed (see supplemental materials, available at www.jneurosci.org, for details).

\section{Results}

\section{Behavioral results}

Mean reaction times of the four experimental conditions (TPpainful, TP-nonpainful, TL-painful, TL-nonpainful) were $1014 \pm 135,1025 \pm 115,1022 \pm 109$, and $985 \pm 138$ ms (mean \pm SD), respectively (Fig. $2 A$ ). There was no significant main effect of task $(F<1)$ or stimulus $(F<1)$, and there was a marginally significant interaction $\left(F_{(1,17)}=3.65, p=0.07\right)$. Post hoc comparisons showed that there was no significant RT difference between judging pain of painful and nonpainful stimuli (TPpainful vs TP-nonpainful; $p>0.05$ ); however, judging laterality of painful stimuli was significantly slower than judging nonpainful stimuli (TL-painful vs TL-nonpainful; $p<0.05$ ). This Stroop-type effect (Stroop, 1935) suggests that painful stimuli might attract attention and interfere with laterality judgment even though the painful aspect of stimuli was unrelated to task requirement.

Accuracy for TP-painful, TP-nonpainful, TL-painful, and TL-nonpainful was $93 \pm 6 \%, 95 \pm 4 \%, 94 \pm 6 \%$, and $96 \pm 3 \%$, respectively (Fig. $2 \mathrm{~B}$ ). There was a small but significant stimulus effect $\left(1 \%, F_{(1,17)}=7.15, p=0.016\right)$. Painful stimuli had a lower 

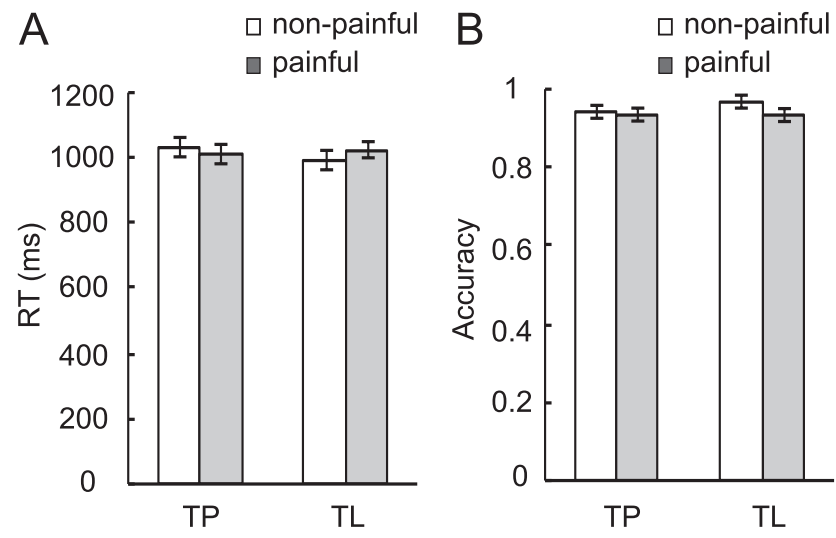

Figure 2. Behavioral results. $A$, RT for four experimental conditions. There was no significant main effect of task or stimulus; the task-by-stimulus interaction was marginally significant ( $p=0.07$ ). $B$, Accuracy for four experimental conditions. There was a small but significant stimulus but not task effect; the main effect of stimulus and the interaction were not significant.

accuracy than nonpainful stimuli. There was no significant main effect of the task $\left(F_{(1,17)}=1.35, p=0.26\right)$. The interaction was not significant $(F<1)$.

\section{fMRI results}

ROI analysis

We performed ROI analysis on FI and ACC based on the activation of these two regions in all experimental conditions compared with baseline fixation (Fig. 3A, Table 1). The coordinates of the ROIs were as follows: ACC, centered at [0 846 ; ; bilateral FI, centered at [ $-3224-4]$ and [34 24 4].

Parameter estimates of the four experimental conditions were extracted from FI and ACC ROIs (Fig. 3B) and entered into a ACC), task (TP, TL), and stimulus (painful, nonpainful). There was no significant effect of task $(F<1)$; there was a marginally significant effect of stimulus $\left(F_{(1,17)}=3.47, p=0.08\right)$; there was a significant main effect of ROI $\left(F_{(1,17)}=80.94, p<0.001\right)$; there was a significant ROI-by-stimulus interaction $\left(F_{(1,17)}=5.79, p<\right.$ $0.05)$; none of the other interactions were significant $(F<1)$. We further examined the effect of stimulus within each ROI averaged across tasks. Planned comparisons showed that the differential activation between painful and nonpainful stimuli reached significance for FI $\left(F_{(1,17)}=12.39, p<0.01\right)$ but not for ACC $(F<1)$. These findings suggest that neural activity of FI is more sensitive to painful stimuli than that of ACC regardless of whether subjects were explicitly asked to judge others' pain, which may imply a more specific role of FI than ACC in empathy for others' pain. three-way ANOVA model with the following factors: ROI (FI,

Table 1. Brain activations averaged across all four experimental conditions

\begin{tabular}{|c|c|c|c|c|c|c|}
\hline \multirow[b]{2}{*}{ Region } & \multirow[b]{2}{*}{$\mathrm{BA}$} & \multicolumn{3}{|c|}{ MNI coordinates } & \multirow[b]{2}{*}{ Z } & \multirow[b]{2}{*}{$k$} \\
\hline & & $x$ & $y$ & $z$ & & \\
\hline R fusiform gyrus & 37 & 32 & -54 & -10 & 7.73 & 29,123 \\
\hline R superior occipital gyrus & 18 & 20 & -94 & 6 & 7.46 & \\
\hline L middle occipital gyrus & 18 & -30 & -88 & 14 & 7.12 & \\
\hline L fusiform gyrus & 37 & -32 & -58 & -16 & 6.89 & \\
\hline LSII & 3 & -56 & -20 & 22 & 5.40 & \\
\hline LSI & 3 & -58 & -24 & 42 & 5.40 & \\
\hline Bilateral ACC & 32 & 0 & 8 & 46 & 6.5 & 1025 \\
\hline R frontal operculum & 44 & 48 & 10 & 30 & 4.44 & 929 \\
\hline R somatomotor cortex & 6 & 26 & -6 & 52 & 4.25 & \\
\hline L insula* & & -32 & 24 & 4 & 4.29 & 119 \\
\hline R frontal operculum & 47 & 48 & 20 & -6 & 4.07 & 194 \\
\hline R insula & & 34 & 24 & 4 & 3.17 & \\
\hline
\end{tabular}

$P_{\text {false discovery rate }}<0.05$, voxel size $=2 \times 2 \times 2 \mathrm{~mm}$. SI, Primary somatosensory cortex; Sll, secondary somatosensory cortex; $B A$, Brodmann area; $R$, right; $L$, left. * Cluster-level $p=0.08$ uncorrected. These clusters cover regions in the frontoparietal network.

To examine whether there is a laterality effect of bilateral FI activation, we also conducted an ANOVA with left FI and right FI as separate ROIs (Fig. 3C). There was no significant main effect of task $(F<1)$ or laterality $\left(F_{(1,17)}=1.45, p>0.05\right)$; the main effect of stimulus was still significant $\left(F_{(1,17)}=12.39, p<0.01\right)$. There was a significant laterality-by-stimulus interaction $\left(F_{(1,17)}=5.57\right.$, $p=0.03)$, indicating that the increased activation for painful stimuli was greater in left FI than in right FI. None of the other interactions were significant $(F<1)$. Planned comparisons were then conducted to examine the differential activation for painful and nonpainful stimuli (pooled over tasks) for left FI and right FI separately; this difference in activation reached significance for both left FI $\left(F_{(1,17)}=23.36, p<0.001\right)$ and right FI $\left(F_{(1,17)}=\right.$ 4.84, $p<0.05)$.

\section{Whole-brain analysis}

We also performed whole-brain analysis to verify our findings from the ROI analysis. We directly compared the brain activation maps of painful and nonpainful stimuli averaged across tasks. Increased activation for painful stimuli compared with nonpainful stimuli was found in bilateral FI but not in ACC (Fig. 4A, Table 2). Other brain regions that showed significant activation for painful stimuli include bilateral primary and secondary somatosensory cortex, somatomotor cortex, superior medial prefrontal cortex (smPFC), presupplementary motor area (preSMA), occipitotemporal visual areas, cerebellum, and thalamus (Table 2). We also list regions that showed less activation for painful than nonpainful stimuli. These include visual occipitotemporal cortices, middle and superior prefrontal cortices, subgenual cingulate cortex, putamen, and caudate nucleus.

No activation difference reached sig-
A

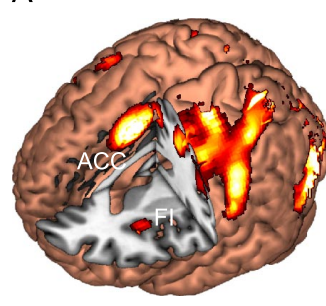

B

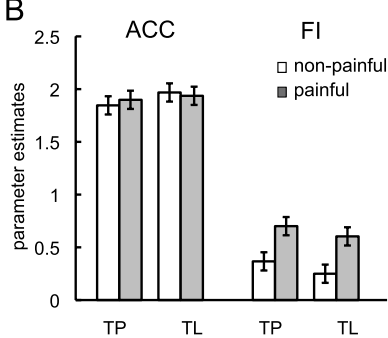

C

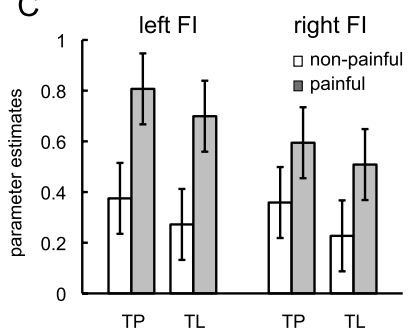

Figure 3. ROl analysis of the parameter estimates of ACC and FI for four experimental conditions (TP-nonpainful, TP-painful, TL-nonpainful, TL-painful). A, Localization of ACC and FI ROls derived from activations common to all four experimental conditions. $\boldsymbol{B}$, ACC showed comparable activation levels to all four conditions; Fl showed significant increased activation for painful compared with nonpainful stimuli independent of the task. This ROl-by-stimulus interaction was significant (see Results for details). C, Responses in left Fl and right Fl separately. Error bars represent 95\% confidence intervals. nificance for the contrast between TP and TL (Fig. 4B), or the task-by-stimulus interaction effects TP (painful $>$ nonpainful $)>$ TL (painful $>$ nonpainful) and TL $($ painful $>$ nonpainful $)>\mathrm{TP}($ painful $>$ nonpainful). Pairwise comparisons between painful and nonpainful stimuli under TP (painful $>$ nonpainful) yielded significant activation in FI bilaterally, and somatosensory, superior parietal, and occipitotemporal visual areas, but not in ACC (Fig. 4C, Table 3). Pairwise comparison between painful and nonpainful stimuli under TL (painful > 


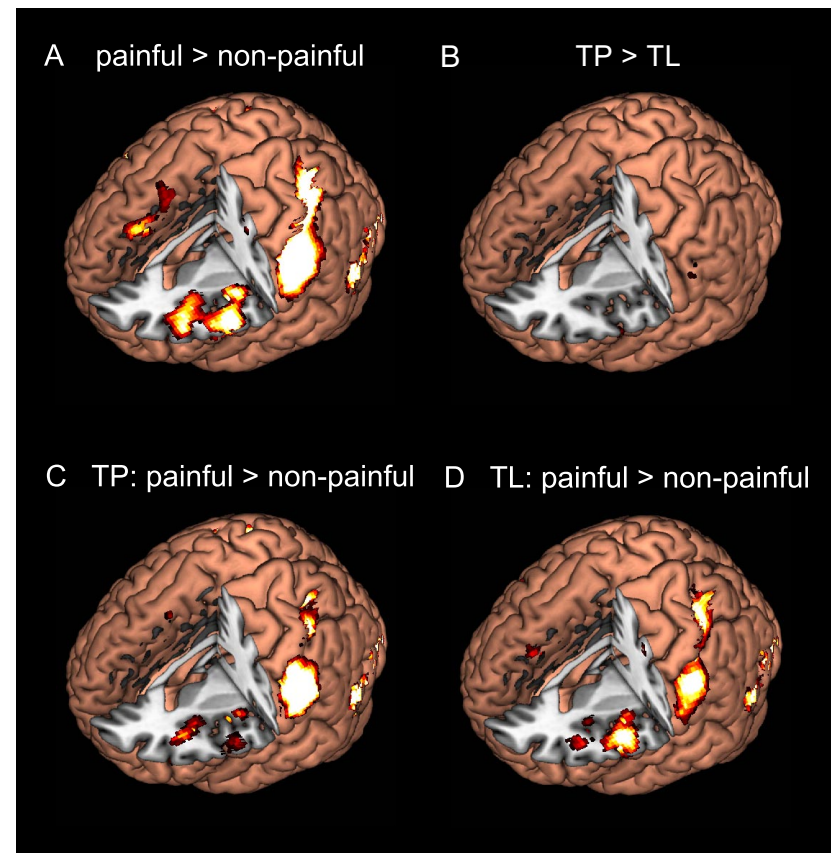

Figure 4. Whole-brain analysis. A, Stimulus effect: painful versus nonpainful stimuli (averaged across tasks). $\boldsymbol{B}$, Task effect: TP versus TL. C, Pairwise comparison: judging pain of painful stimuli versus nonpainful stimuli. $\boldsymbol{D}$, Pairwise comparison: judging laterality of painful stimuli versus nonpainful stimuli. $\mathrm{Fl}$, but not $\mathrm{ACC}$, showed a significant increase in activation for painful stimuli regardless of the task.

nonpainful) also yielded significant activation in FI bilaterally rather than ACC (Fig. 4D). Other activation patterns are listed in Table 3.

\section{Discussion}

Our main findings are twofold. First, FI robustly responded to the sight of others' pain bilaterally, regardless of whether the observer was explicitly asked to evaluate pain. Second, ACC activation did not differentiate between painful and nonpainful stimuli, or between pain judgment and laterality judgment; that is, increase in activation due to empathy for pain was significantly greater in FI than in ACC. These findings showed a clear functional dissociation between FI and ACC in the process of empathizing with others' pain.

\section{A unique role of FI in empathy for pain}

Our data indicated that we showed that FI, instead of ACC, differentially responded to the sight of others' pain, regardless of whether subjects needed to empathize intentionally with others, indicating that FI may be functionally more specific than ACC in processing empathetic responses without effortful thought about or evaluation of other people's situations. FI has been traditionally conceived of as a limbic sensory region that integrates multimodal sensory information. It has been recently proposed that FI is able to translate sensory information into conscious awareness of bodily sensations and emotional feelings (Critchley et al., 2004; Craig, 2009). Our findings support this view by showing that FI is consistently active when painful feelings are elicited regardless of the cognitive requirement of the task, which distinguishes FI from other brain regions (such as ACC) that are traditionally considered to process empathy for pain.

Another important aspect of our observation is the automaticity of FI recruitment in processing empathy. Our data indicate that even when subjects' attention is not explicitly focused on
Table 2. Whole-brain analysis: stimulus effects

\begin{tabular}{|c|c|c|c|c|c|c|}
\hline \multirow[b]{2}{*}{ Region } & \multirow[b]{2}{*}{$\mathrm{BA}$} & \multicolumn{3}{|c|}{ MNI coordinates } & \multirow[b]{2}{*}{ Z } & \multirow[b]{2}{*}{$k$} \\
\hline & & $x$ & $y$ & $z$ & & \\
\hline \multicolumn{7}{|l|}{ Painful $>$ nonpainful } \\
\hline L middle occipital gyrus & 37 & -44 & -64 & 0 & 6.54 & 2771 \\
\hline L inferior occipital gyrus & 19 & -44 & -74 & -6 & 6.36 & \\
\hline R middle occipital gyrus & 18 & 34 & -84 & 4 & 5.79 & 2194 \\
\hline R inferior temporal gyrus & 37 & 54 & -58 & -2 & 5.57 & \\
\hline R inferior occipital gyrus & 18 & 26 & -90 & -4 & 5.44 & \\
\hline $\mathrm{LSI} / \mathrm{SII}$ & 2 & -56 & -30 & 38 & 6.08 & 2878 \\
\hline L superior parietal lobule & 7 & -38 & -46 & 58 & 5.70 & \\
\hline L inferior parietal lobule & 40 & -34 & -42 & 44 & 4.84 & \\
\hline R superior parietal lobule & 7 & 26 & -54 & 58 & 5.25 & 2032 \\
\hline $\mathrm{RSI} / \mathrm{SII}$ & 3 & 56 & -22 & 40 & 5.19 & \\
\hline L somatomotor cortex & 6 & -48 & 4 & 22 & 4.92 & 2942 \\
\hline L frontal operculum & $44 / 47$ & -52 & 12 & 6 & 4.46 & \\
\hline Linsula & & -36 & 22 & -2 & 4.39 & \\
\hline R frontal operculum & $44 / 47$ & 52 & 12 & 8 & 4.81 & 1423 \\
\hline R somatomotor cortex & 6 & 54 & 8 & 28 & 4.40 & \\
\hline R superior temporal pole & 38 & 40 & 12 & -20 & 4.32 & 105 \\
\hline Rinsula & & 32 & 14 & -20 & 3.27 & \\
\hline L superior medial frontal gyrus & 8 & -4 & 38 & 46 & 4.00 & 478 \\
\hline Presupplementary motor area & 8 & 0 & 20 & 54 & 3.17 & \\
\hline L superior occipital gyrus & 19 & -24 & -70 & 34 & 3.86 & 89 \\
\hline L cerebellum & & 20 & -76 & -22 & 3.70 & 97 \\
\hline L thalamus & & -14 & -8 & -12 & 3.55 & 106 \\
\hline \multicolumn{7}{|l|}{ Nonpainful > painful } \\
\hline R lingual gyrus & 18 & 12 & -72 & -6 & 5.48 & 7471 \\
\hline Rprecuneus & $18 / 19$ & 2 & -40 & 50 & 4.95 & \\
\hline R angular gyrus & 39 & 42 & -62 & 30 & 4.95 & 1257 \\
\hline R superior temporal gyrus & 41 & 44 & -34 & 20 & 4.65 & 342 \\
\hline R middle frontal gyrus & 8 & 24 & 28 & 48 & 4.58 & 635 \\
\hline R superior frontal gyrus & 9 & 26 & 44 & 38 & 4.00 & \\
\hline L angular gyrus & $7 / 39$ & -34 & -68 & 7 & 4.14 & 457 \\
\hline L middle occipital gyrus & 39 & -36 & -62 & 30 & 3.51 & \\
\hline L superior temporal gyrus & 42 & -52 & -40 & 16 & 4.14 & 311 \\
\hline L rolandic operculum & & -46 & -16 & 16 & 3.13 & 76 \\
\hline L rolandic operculum & & -62 & -6 & 14 & 4.05 & 79 \\
\hline L subgenual cingulate cortex & 25 & -4 & 34 & 0 & 3.59 & 209 \\
\hline L middle temporal gyrus & 22 & -58 & -16 & 2 & 3.36 & 192 \\
\hline L putamen & & -18 & 10 & -4 & 3.95 & 147 \\
\hline L caudate nucleus & & -6 & 18 & -6 & 3.69 & \\
\hline
\end{tabular}

$P_{\text {false discovery rate }}<0.05$, voxel size $=2 \times 2 \times 2 \mathrm{~mm}$. SI, Pprimary somatosensory cortex; SIl, secondary somatosensory cortex; $B A$, Brodmann area; $R$, right; $L$, left.

others' pain, empathetic responses in FI still occur to the same extent as when subjects are explicitly evaluating pain, suggesting that FI activation in response to the sight of others' pain can be an automatic process, in which top-down modulation may not necessarily be required. This automaticity of FI activation provides a neural mechanism for the perception-action model of empathy, which states that the sight of another person's emotional state directly and immediately elicits a mental representation of that state in the observer (Preston and de Waal, 2002). Considering the interoceptive function of the insula (Critchley et al., 2004; Craig, 2009), this process can be achieved through a direct mapping of another person's physiological state to one's own internal state in FI and its interconnected sensory regions (Carr et al., 2003; Singer et al., 2009). This finding is also in accordance with the late appraisal model of empathy (de Vignemont and Singer, 2006), in that empathetic responses occur immediately after emotional cues (increased FI activation for painful stimuli regardless of the cognitive task), accompanied by the appraisal process in parallel (equal yet significant increment in ACC activation for each experimental condition compared with fixation). In this context, the present study shows that FI, but not ACC, is specialized in processing empathy for others' pain and that FI activation 
Table 3. Pairwise comparisons of brain activation between experimental conditions

\begin{tabular}{|c|c|c|c|c|c|c|}
\hline \multirow[b]{2}{*}{ Region } & \multirow[b]{2}{*}{ BA } & \multicolumn{3}{|c|}{ MNI coordinates } & \multirow[b]{2}{*}{ Z } & \multirow[b]{2}{*}{$k$} \\
\hline & & $x$ & $y$ & $z$ & & \\
\hline \multicolumn{7}{|l|}{ TP (painful > nonpainful) } \\
\hline L middle temporal gyrus & 37 & -44 & -64 & -2 & 7.09 & 2035 \\
\hline L inferior occipital gyrus & 19 & -46 & -72 & -6 & 6.27 & \\
\hline L middle occipital gyrus & 18 & -28 & -94 & 0 & 5.38 & \\
\hline R inferior temporal gyrus & 37 & 54 & -60 & -4 & 5.64 & 1551 \\
\hline R middle occipital gyrus & 18 & 36 & -86 & 6 & 5.17 & \\
\hline LSI/SII & 2 & -56 & -30 & 34 & 5.64 & 1739 \\
\hline L superior parietal gyrus & 40 & -40 & -44 & 58 & 4.41 & \\
\hline R superior parietal gyrus & 7 & 26 & -52 & 58 & 5.36 & 1557 \\
\hline RSI & 2 & 42 & -38 & 58 & 5.14 & \\
\hline R inferior parietal gyrus & 7 & 30 & -48 & 52 & 5.14 & \\
\hline R frontal operculum & 44 & 52 & 10 & 24 & 4.58 & 373 \\
\hline R superior temporal pole & 38 & 40 & 10 & -18 & 4.22 & 98 \\
\hline R frontal operculum & 38 & 32 & 18 & -20 & 3.19 & \\
\hline R middle temporal gyrus & 21 & 48 & 4 & -20 & 3.15 & \\
\hline L somatomotor cortex & 6 & -48 & 4 & 24 & 4.11 & 499 \\
\hline L frontal operculum & 6 & -60 & 10 & 12 & 3.52 & \\
\hline Linsula & & -34 & 20 & 2 & 4.03 & 467 \\
\hline Linsula & & -38 & -2 & 0 & 3.91 & 136 \\
\hline R frontal operculum & 47 & 40 & 32 & -8 & 3.90 & 180 \\
\hline \multicolumn{7}{|l|}{ TL (painful > nonpainful) } \\
\hline L inferior occipital gyrus & 19 & -44 & -72 & -6 & 5.48 & 2075 \\
\hline L middle occipital gyrus & 18 & -32 & -92 & 8 & 5.38 & \\
\hline R middle occipital gyrus & 18 & 34 & -86 & 8 & 5.12 & 1496 \\
\hline R inferior temporal gyrus & 19 & 46 & -72 & -8 & 4.97 & \\
\hline L frontal operculum & & -50 & 10 & 6 & 4.82 & 1425 \\
\hline L somatomotor cortex & 6 & -50 & 2 & 28 & 4.01 & \\
\hline L superior parietal gyrus & 2 & -32 & -46 & 58 & 4.70 & 2116 \\
\hline L inferior parietal gyrus & 2 & -42 & -34 & 44 & 4.70 & \\
\hline R superior parietal gyrus & 7 & 24 & -54 & 56 & 4.48 & 721 \\
\hline R somatosensory cortex & 3 & 52 & -22 & 38 & 3.84 & \\
\hline R frontal operculum & & 50 & 10 & 6 & 4.22 & 198 \\
\hline L superior medial frontal gyrus & 8 & -4 & 32 & 44 & 3.65 & 97 \\
\hline R somatomotor cortex & 44 & 54 & 8 & 28 & 3.37 & 119 \\
\hline
\end{tabular}

$P_{\text {false discovery rate }}<0.05$, voxel size $=2 \times 2 \times 2 \mathrm{~mm}$. SI, Primary somatosensory cortex; Sll, secondary somatosensory cortex; $B A$, Brodmann area; $R$, right; $L$, left.

in response to pain empathy can be elicited automatically, independent from the explicit task requirement. In addition, we conducted PPI analysis (see supplemental materials, available at www.jneurosci.org) to explore how the context of empathy for painful stimuli might modulate the functional connectivity between the seed regions, namely ACC and FI, and other brain regions. Under the context of painful stimuli, FI showed significant decrease in functional connectivity primarily with SMA, smPFC, and inferior frontal gyrus, which have been suggested to participate in mentalizing and empathetic processes (Gallagher et al., 2000; Carr et al., 2003; Decety and Chaminade, 2003; Gu and Han, 2007; Akitsuki and Decety, 2009) and are considered part of the "social brain" (Blakemore, 2008). As a key region in the pain matrix, FI works with a network of brain regions within and outside of the pain matrix to convey the awareness of another individual's emotional state to generate isomorphic feelings in oneself.

\section{Revisiting the role of ACC in pain and empathy}

In contrast to significant increase in FI activation by pain, ACC activation was not significantly increased for the sight of others' pain (painful vs nonpainful stimuli) or the evaluation of others' situation (TP vs TL) in our experiment, suggesting that ACC may not be specific for either automatic or controlled empathetic responses. Although ACC activation has been considered as a neurobiological marker of empathy for pain in previous studies, the role of ACC in general information processing has rarely been taken into account, which is confounded with empathy processing. Therefore, as a key manipulation in our design, we purposely equated cognitive loads for the empathy task (TP) and the control task (TL) as indexed by comparable RTs. Hence, although our findings appear to conflict with previous studies that reported involvement of ACC in empathy, they support the idea that ACC may not be specific for empathy, but rather participate in this process through its general role in voluntary control of behaviors. For instance, ACC is involved in perspective taking (Ruby and Decety, 2004; Lamm et al., 2007), which is an important process in empathy. It is noteworthy that, although empathy for pain did not activate dorsal ACC, it did activate pre-SMA, together with the cerebellum, which supports the view that pain often triggers motor preparation as well as inhibition, or "fight or flight" responses to pain, that possess great survival significance (Peyron et al., 2000). Under the context of empathy for pain, the functional connectivity between ACC and visual attention areas (occipitotemporal cortex and brain areas along the intraparietal sulcus) was significantly decreased, as revealed by the PPI analysis (see supplemental material, available at www.jneurosci.org, for details), suggesting that the involvement of ACC in empathy for pain might be implemented through its role in visual attention and response anticipation.

\section{Functional dissociation between FI and ACC}

Increase in FI activation without the concurrent increase in ACC activation in the current study not only clarifies our understanding about differential roles of each region subserved in pain empathy, but also elucidates their discrepancy in general cognitive processes. Most studies on the roles of FI and ACC in cognitive function have focused on the coactivation of these two regions in cognitively demanding tasks and the coordination of these two regions in processing salience (Seeley et al., 2007; Sridharan et al., 2008). However, it is worth noting that a recent study by Sridharan et al. (2008) suggested that FI, rather than ACC, serves as the causal outflow hub at the junction of the central executive network and default mode network by showing that right FI had the highest number of causal outflow connections, the lowest number of causal inflow connections, and the shortest path lengths among all regions tested in a Granger causality analysis. The authors proposed that right FI has a strong causal influence on ACC and may generate signals to trigger hierarchical voluntary control. It is likely that in the empathy for pain paradigm, FI initially identifies the most homeostatically significant input of pain and integrates highly interoceptive information with outer-world representations in order for ACC to transform this evaluative signal into voluntary control over behavioral decisions (Craig, 2009). We showed for the first time that when cognitive load is carefully matched between painful and nonpainful conditions, FI, but not ACC, specifically responds to empathy for pain, suggesting a more direct and essential role of FI than ACC in processing empathy for pain. This finding challenges the current consensus that ACC is indispensable in empathetic responses and singles out the importance of FI in empathetic processes.

\section{References}

Akitsuki Y, Decety J (2009) Social context and perceived agency affects empathy for pain: an event-related fMRI investigation. Neuroimage 47: $722-734$.

Blakemore SJ (2008) The social brain in adolescence. Nat Rev Neurosci 9:267-277.

Carr L, Iacoboni M, Dubeau MC, Mazziotta JC, Lenzi GL (2003) Neural 
mechanisms of empathy in humans: a relay from neural systems for imitation to limbic areas. Proc Natl Acad Sci U S A 100:5497-5502.

Cohen RA, Kaplan RF, Zuffante P, Moser DJ, Jenkins MA, Salloway S, Wilkinson H (1999) Alteration of intention and self-initiated action associated with bilateral anterior cingulotomy. J Neuropsychiatry Clin Neurosci 11:444-453.

Cohen RA, Paul R, Zawacki TM, Moser DJ, Sweet L, Wilkinson H (2001) Emotional and personality changes following cingulotomy. Emotion $1: 38-50$.

Craig AD (2009) How do you feel-now? The anterior insula and human awareness. Nat Rev Neurosci 10:59-70.

Craig AD, Chen K, Bandy D, Reiman EM (2000) Thermosensory activation of insular cortex. Nat Neurosci 3:184-190.

Critchley HD (2004) The human cortex responds to an interoceptive challenge. Proc Natl Acad Sci U S A 101:6333-6334.

Critchley HD, Wiens S, Rotshtein P, Ohman A, Dolan RJ (2004) Neural systems supporting interoceptive awareness. Nat Neurosci 7:189-195.

Davis KD, Hutchison WD, Lozano AM, Dostrovsky JO (1994) Altered pain and temperature perception following cingulotomy and capsulotomy in a patient with schizoaffective disorder. Pain 59:189-199.

Decety J, Chaminade T (2003) Neural correlates of feeling sympathy. Neuropsychologia 41:127-138.

de Vignemont F, Singer T (2006) The empathic brain: how, when and why? Trends Cogn Sci 10:435-441.

Eckert MA, Menon V, Walczak A, Ahlstrom J, Denslow S, Horwitz A, Dubno JR (2009) At the heart of the ventral attention system: the right anterior insula. Hum Brain Mapp 30:2530-2541.

Fan J, Hof PR, Guise KG, Fossella JA, Posner MI (2008) The functional integration of the anterior cingulate cortex during conflict processing. Cereb Cortex 18:796-805.

Friston KJ, Frith CD, Turner R, Frackowiak RSJ (1995) Characterizing evoked hemodynamics with fMRI. Neuroimage 2:157-165.

Friston KJ, Fletcher P, Josephs O, Holmes A, Rugg MD, Turner R (1998) Event-related fMRI: characterizing differential responses. Neuroimage $7: 30-40$.

Gallagher HL, Happé F, Brunswick N, Fletcher PC, Frith U, Frith CD (2000) Reading the mind in cartoons and stories: an fMRI study of "theory of mind" in verbal and nonverbal tasks. Neuropsychologia 38:11-21.

Genovese CR, Lazar NA, Nichols T (2002) Thresholding of statistical maps in functional neuroimaging using the false discovery rate. Neuroimage 15:870-878.

Gu X, Han S (2007) Attention and reality constraints on the neural processes of empathy for pain. Neuroimage 36:256-267.

Hahn B, Ross TJ, Stein EA (2007) Cingulate activation increases dynamically with response speed under stimulus unpredictability. Cereb Cortex 17:1664-1671.

Jabbi M, Swart M, Keysers C (2007) Empathy for positive and negative emotions in the gustatory cortex. Neuroimage 34:1744-1753.
Jackson PL, Meltzoff AN, Decety J (2005) How do we perceive the pain of others? A window into the neural processes involved in empathy. Neuroimage 24:771-779.

Kennerley SW, Walton ME, Behrens TE, Buckley MJ, Rushworth MF (2006) Optimal decision making and the anterior cingulate cortex. Nat Neurosci 9:940-947.

Lamm C, Batson CD, Decety J (2007) The neural substrate of human empathy: effects of perspective-taking and cognitive appraisal. J Cogn Neurosci 19:42-58.

Naito E, Kinomura S, Geyer S, Kawashima R, Roland PE, Zilles K (2000) Fast reaction to different sensory modalities activates common fields in the motor areas, but the anterior cingulate cortex is involved in the speed of reaction. J Neurophysiol 83:1701-1709.

Peyron R, Laurent B, García-Larrea L (2000) Functional imaging of brain responses to pain: a review and meta-analysis. Neurophysiol Clin 30:263-288.

Preston SD, de Waal FB (2002) Empathy: its ultimate and proximate bases. Behav Brain Sci 25:1-20; discussion 20-71.

Rainville P, Duncan GH, Price DD, Carrier B, Bushnell MC (1997) Pain affect encoded in human anterior cingulate but not somatosensory cortex. Science 277:968-971.

Ruby P, Decety J (2004) How would you feel versus how do you think she would feel? A neuroimaging study of perspective-taking with social emotions. J Cogn Neurosci 16:988-999.

Rudebeck PH, Buckley MJ, Walton ME, Rushworth MF (2006) A role for the macaque anterior cingulate gyrus in social valuation. Science 313:1310-1312.

Seeley WW, Menon V, Schatzberg AF, Keller J, Glover GH, Kenna H, Reiss AL, Greicius MD (2007) Dissociable intrinsic connectivity networks for salience processing and executive control. J Neurosci 27:2349-2356.

Singer T, Seymour B, O’Doherty J, Kaube H, Dolan RJ, Frith CD (2004) Empathy for pain involves the affective but not sensory components of pain. Science 303:1157-1162.

Singer T, Critchley HD, Preuschoff K (2009) A common role of insula in feelings, empathy and uncertainty. Trends Cogn Sci 13:334-340.

Sridharan D, Levitin DJ, Menon V (2008) A critical role for the right frontoinsular cortex in switching between central-executive and default-mode networks. Proc Natl Acad Sci U S A 105:12569-12574.

Stroop JR (1935) Studies of interference in serial verbal reactions. J Exp Psych 18:643-662.

Wager TD, Rilling JK, Smith EE, Sokolik A, Casey KL, Davidson RJ, Kosslyn SM, Rose RM, Cohen JD (2004) Placebo-induced changes in fMRI in the anticipation and experience of pain. Science 303:1162-1167.

Wicker B, Keysers C, Plailly J, Royet JP, Gallese V, Rizzolatti G (2003) Both of us disgusted in My insula: the common neural basis of seeing and feeling disgust. Neuron 40:655-664. 